\title{
El Centro del Poder para los Reyes de Copán
}

Loa Traxler

\section{Resumen}

La Acrópolis de Copán representa el centro del poder para los reyes de esta comunidad Clásica maya, el punto para sus actividades diarias, eventos especiales y su lugar de descanso final. El ancla del poder político y religioso durante cuatro siglos; la Acrópolis conserva la historia dinámica de la expresión del poder real, ejercido por la élite. Su arquitectura refleja el uso de la monumentalidad y de la imaginería real para poner a la élite aparte de la población común tanto física como metafóricamente. Siendo central al paisaje de la comunidad, la Acrópolis combinaba manifestaciones públicas con precintos exclusivos para la familia real y para las actividades palaciegas. La evolución de la Acrópolis y los restos asociados de la nobleza y de sus vidas nos cuentan la historia en cuanto a la afirmación del poder real y del esfuerzo constante por reforzarlo dentro del reinado.

Palabras Clave: Copán, poder político y religioso, monumentalidad, imaginería real.

\section{Abstract}

The Acropolis of Copan represented the center of power for the kings of this Classic maya community, the locus of daily activities, special events and place of final rest. The anchor of political and religious power during four centuries; the Acropolis preserves the dynamic history of royal power expression executed by the elite. Its architecture reflects the use of monumentality and regal imagery to set the elite apart from the common population both physically and metaphorically. Central to the community's landscape, the Acropolis combined public manifestations with exclusive enclosures for the royal family and the palatial activities. The evolution of the Acropolis, and the remains associated with royalty and their lives, tell us a story in terms of affirmation of royal power and the constant effort to reinforce it within the reign. 
Keywords: Copan, political and religious power, monumentality, royal imagery.

Loa Traxler (Iptraxler@unm.edu), Universidad de Nuevo México (University of New Mexico). 


\section{INTRODUCCIÓN}

El asentamiento humano del valle de Copán comenzó durante el segundo milenio A.C., aunque la arquitectura más temprana conservada dentro del sitio mismo de Copán fecha de aproximadamente los 100-400 D.C. Estas estructuras tempranas en Copán representan tradiciones regionales de construcción de tierra y guijarros, a las que se les agregaba técnicas bruscas de albañilería y de argamasa de cal. En su mayoría, los restos tempranos arquitectónicos en Copán se encuentran al sur y al oeste del Grupo Principal, debajo de la arquitectura del período Clásico en la Gran Plaza y en la Acrópolis, y debajo del Patio A de Las Sepulturas (Hall y Viel 2004; Cheek 1983; Cheek y Kennedy Embree 1983; Fash 1983). La arquitectura temprana en Copán refleja una continuidad de las tradiciones constructivas documentadas para el período Preclásico Tardío (ca. 300 A.C.-250 D.C.) en otros sitios del valle de Copán y en la más extensa cuenca del Río Copán (Fash y Fash 2000; Maca 2002; Canuto 2002, 2004).

Todavía queda por definirse la naturaleza y la extensión del asentamiento durante el Preclásico Tardío en Copán, pero la presencia de por lo menos una estructura temprana marcada ritualmente, la Estructura 10L-sub-1 (Cheek 1983), sugiere una instalación ceremonial, ubicada originalmente debajo de la Gran Plaza. Textos retrospectivos sugieren la importancia de este asentamiento temprano. Estos textos se esculpieron durante el período Clásico Tardío, que mencionan el fin de período del K'atun 8.6.0.0.0 en el año 159 D.C. Una fecha subsiguiente de 8.6.0.10.8 (el 160 D.C.) en la Estela I de Copán está asociada con un pasaje destruido que incluye el símbolo jeroglíico para la cabeza del murciélago, que posteriormente se convirtió en el Glifo Emblema de Copán (Stuart 2004). Los restos de asentamientos contemporáneos que se han documentado en otros lugares del valle indican que la población local incluía subgrupos con élites y rangos sociales durante los períodos del Preclásico Tardío y Clásico Temprano, antes de la fundación de la dinastía (Fash 2001).

\section{Establecimiento de la Dinastía en Copán}

Durante la parte temprana del siglo $V$ en Copán, los textos jeroglíícos marcan la importancia de un individuo llamado K'inich Yax K'uk' Mo'. A este rey constantemente se le mencionaba en textos con títulos únicos, incluyendo los de "Fundador" y "Señor del Oeste". El texto retrospectivo sobre el Altar Q, esculpido durante el período 
Clásico Tardío, unos 350 años después del establecimiento dinástico, recordó la accesión al poder y la llegada de K'inich Yax K'uk' Mo' a Copán. K'inich Yax K'uk' Mo' y sus quince sucesores aparecen en los lados del Altar $Q$ y juntos representan el período dinástico del Clásico en Copán. La llegada al poder de K'inich Yax K'uk' Mo' en el año 426 D.C. estableció su puesto como líder tanto político como religioso de la comunidad de Copán, mientras que su participación en ceremonias que se celebraron para el gran fin de período del calendario para el año 9.0.0.0.0 (el 435 D.C.) solidificó su control sobre la comunidad y su lugar en la historia de ésta. Aunque la línea directa de sucesión desde K'inich Yax K'uk' Mo' y a través de los quince reyes hasta Yax Pasaj Chan Yopaat (gobierno 763- ca. 820 D.C.) ha sido tema de debate (e.g. Viel 1999), la integridad de la línea de sucesión, en concepto y en convención, ya se definió por medio de los textos históricos de Copán (Martin y Grube 2000: 191-213). Los retratos de los gobernantes en orden de sucesión en el Altar $Q$ refuerzan la idea de la culminación de línea dinástica en el período Clásico Tardío, mostrando al decimosexto gobernante aceptando el símbolo del puesto de parte de su ancestro, K'inich Yax K'uk' Mo'.

La forma en que K'inich Yax K'uk' Mo' estableció control sobre la comunidad de Copán, temprano en el quinto siglo, permanece sin aclarar. Las expresiones específicas en el texto del Altar $Q$ sobre su llegada por lo general denotan la toma de una comunidad, según Stuart (2004). La accesión y la llegada de K'inich Yax K'uk' Mo' se mencionó de nuevo en monumentos del período Clásico Tardío en el sitio subsidiario de Qluiriguá en forma paralela al texto del Altar Q (Looper 1999). Estas inscripciones registran el dominio de Yax K'uk' Mo' sobre un gobernante local de Quiriguá y proveen la evidencia que este centro en el valle del Motagua se convirtió en parte de la comunidad de Copán durante el Clásico Temprano.

\section{ARQUITECTURADEL CLÁSICO TEMPRANO EN LANUEVACAPITALDE COPÁN}

Las excavaciones en la Acrópolis de Copán han documentado la arquitectura y los monumentos asociados con el reino de K'inich Yax K'uk' Mo' y de sus sucesores inmediatos (Sharer et al. 1999), verificando y amplificando las descripciones históricas sobre Copán y Quiriguá. Las excavaciones que condujo el Programa de Investigación de la Acrópolis Temprana (PIAT) del Museo de la Universidad de Pensilvania, dirigido por Robert Sharer, y su antecesor el Proyecto Arqueológico de la Acrópolis de Copán (PAAC), dirigido por William Fash, indican que la disposición 
del centro de la comunidad estaba reconfigurado para servir como la capital de una nueva dinastía, temprano en el siglo $V$ D.C. Los esfuerzos de muchos colegas del PIAT y del más grande PAAC se reflejan en esta presentación. Nuestro trabajo fue posible mediante la colaboración con el Instituto Hondureño de Antropología e Historia y deseo agradecer a su entonces Director Regional, el Profesor Oscar Cruz y a todos los numerosos miembros locales del proyecto quienes a través de los años han abierto túneles, conservado su arquitectura y han ayudado a retomar el rico pasado de Copán.

El complejo arquitectónico que formó el corazón de la nueva capital se componía de una plaza pública grande al norte, un grupo en el centro compuesto por el campo de pelota y un santuario y un grupo palaciego real al sur (Traxler 2001, 2003, 2004a; Fash et al. 2004; Sharer et al. 1999). Este complejo fue el asiento del poder para la comunidad de Copán desde la era de su fundación hasta el fin del gobierno dinástico, cuatro siglos después.

Las investigaciones han demostrado que la expansión de la Gran Plaza fue integral con el plan inicial del período dinástico. Estas investigaciones recientes han modificado reconstrucciones anteriores sobre el desarrollo de la Gran Plaza, basadas por lo general en el importante trabajo de Charles Cheek (1983), durante la primera fase del Proyecto Arqueológico Copán, y antes de él el proyecto de la Institución Carnegie de Washington (Strömsvik 1952). Estructuras de tierra, a las que no se les había reconocido en investigaciones anteriores, ahora son evidentes como la arquitectura más temprana asociada con la Gran Plaza. Estas estructuras de tierra y la estratigrafía de la plaza claramente indican que cualquier área pública que haya estado asociada con la Estr. 10L-sub-1 durante la era predinástica estaba incluida dentro de una Gran Plaza expandida, diseñada y rodeada por arquitectura adicional al momento del establecimiento de la dinastía.

Comenzando en 1986, William y Bárbara Fash, junto con Rudy Larios y sus colegas reiniciaron investigaciones en la famosa Escalinata Jeroglífica, lo que dio nacimiento a la formación del PAAC en 1988. Como parte del proyecto más grande, William Fash dirigió las excavaciones del campo de pelota más temprano de Copán y de los edificios de santuarios adyacentes (ahora parcialmente cubiertos por la Estructura 10L-26 en el lado norte de la Acrópolis), revelando que esta área fue el foco de las ceremonias que marcan el Fin de Período 9.0.0.0.0 y el comienzo del noveno Bak'tun (435-820 D.C.) en el calendario maya (Fash 1998; Fash y Fash 2000; Fash et al. 2004). La excavación de dos monumentos con esta fecha, 
conocidos como el Marcador Motmot y la Estela 63, conectan estas ceremonias de Fin de Período con K'inich Yax K'uk' Mo'. Estos monumentos del Clásico Temprano y la arquitectura asociada con ellos fijaron una secuencia de edificios que culminó con la Estr. 10L-26, dedicada en el período Clásico Tardío. Las gradas con jeroglíficos de la Escalinata Jeroglifica presentan la historia dinástica de Copán y ligan a los sucesores del Clásico Tardío con el Fundador y con el Fin de Período 9.0.0.0.0 (Fash et al. 2004; Stuart 2004). Tanto Fash como Sharer han sugerido que estos eventos de Fin de Período y su asociación cercana con K'inich Yax K'uk' Mo' cimentaron las fortunas de su reinado con las de sus sucesores como los gobernantes divinamente validados del reinado de Copán.

En el extremo sur del complejo arquitectónico, las excavaciones que condujeron Robert Sharer, David Sedat, Alfonso Morales, Julia Miller, Loa Traxler y otros para el PIAT, revelaron un conjunto de patio y posteriormente grupos de plaza, representando el complejo del palacio real, los edificios principales de los reyes del Clásico Temprano de Copán y sus cortes reales (Sharer et al. 1992, 1999, 2005; Sedat y López 2004; Miller sin fecha; Traxler 2001, 2003, 2004b). Las etapas iniciales del complejo de élite incluyeron estructuras de tierra fechadas al principio del siglo V, allá por el tiempo de la fundación de la dinastía y éste fue el punto de partida para el crecimiento de toda la Acrópolis. En el núcleo estaba una plataforma baja, Ilamada Plataforma Yune, excavada en su mayoría por David Sedat, sobre la cual estaba un grupo de patio residencial restringido (Sedat y Lòpez 2004; Sharer et al. 2f005). Deseo reconocer los excelentes dibujos arquitectónicos y esfuerzos de registro realizados por Fernando López, quien ha estado recopilando un sin número de detalles para preparar los dibujos arquitectónicos finales que aparecerán en la serie final de informes tanto para el PIAT como para el PAAC.

La arquitectura sobre la Plataforma Yune se centró en la Estructura Hunal, un edificio de albañilería con una fachada en un estilo distintivo de talud-tablero, característico del México Central. El edificio Hunal, de muchos cuartos, fue destruido en algún momento después de que una recámara de tumba y un entierro se introdujeron debajo de su piso y después de que se habían completado los rituales del entierro. Aunque la evidencia es circunstancial, la Estructura Hunal probablemente sirvió como una residencia real para K'inich Yax K'uk' Mo' durante su reinado y la recámara debajo de este edificio se convirtió en su tumba (Sharer et al. 1999; Sharer 2002). La Plataforma Yune y los edificios posteriores sobre ella formaron el corazón de la Acrópolis temprana, mientras iba desarrollándose durante el período Clásico Temprano. 
Adyacente al grupo residencial real sobre la Plataforma Yune se erguía una serie separada de patios y plazas (Traxler 2001, 2004b). Estos grupos amontonados de patios y plazas se combinan con la arquitectura sobre la Plataforma Yune para formar el complejo del palacio real de Copán (Traxler 2003). Esta arquitectura del Clásico Temprano, ocupada por la más alta élite, se parece con la arquitectura palaciega que se ha encontrado en Tikal, Palenque y en otras capitales mayores mayas (Andrews 1975; Harrison 2003; Christie 2003).

Aunque el primer grupo amontonado se construyó con una arquitectura de tierra y guijarro alrededor del tiempo de la fundación de la dinastía, dentro de unas pocas décadas se transformó con edificios de albañilería arreglados en grupos formales de plazas (Sharer et al. 1992, 1999; Miller sin fecha; Traxler 2001, 2004b). La construcción de los edificios de albañilería en esta área fecha de la parte tardía del reino del Gobernante 2 de Copán (ca. 437-465 D.C.) y su utilización continuó durante los reinos de los siguientes cinco reyes (ca. 465-524 D.C.). A lo largo de la historia de su desarrollo, se mantuvo la forma básica de estas plazas: un grupo de patios rectilíneos e interconectados o plazas definidas por edificios de varios cuartos. Mientras que los detalles del plan del grupo y las interconexiones cambiaron con el paso del tiempo, volviéndose más hacia el interior y con acceso más restringido, la ubicación de estos grupos se conservó durante cuatro fases mayores de construcción.

La evidencia disponible indica que la mayoría de los edificios quizá servían como edificios residenciales de la élite, probablemente para miembros de la familia extensa del gobernante y de sus nobles (Traxler 2001). Las estructuras iniciales de tierra formaron los grupos de patios residenciales de la élite. Los edificios de albañilería, que reemplazaron a las estructuras de tierra, mantuvieron las mismas localidades establecidas y esto, junto con otras líneas de evidencia, sugiere una elaboración de las instalaciones para las mismas actividades residenciales a través del tiempo.

Junto con el palacio real, otras estructuras tempranas dinásticas en Copán exhiben una diversidad de estilos arquitectónicos que servían para mejorar el prestigio y el poder político del rey. Los santuarios ceremoniales asociados con el Campo de Pelota I se construyeron en un estilo elaborado de albañilería característico de la región del Petén (Fash 1998; Fash et al. 2004). Esta región de Tierras Bajas incluía el sitio de Tikal, la capital más poderosa del Clásico Temprano y muy posiblemente cuna de K'inich Yax K'uk' Mo' (Sharer 2003b). En la Plataforma Yune, la albañilería de 
la fachada de la Estructura Hunal incorporó el estilo talud-tablero, típico del México Central, demostrando conexiones con la poderosa capital de Teotihuacan (Fash y Fash 2000; Sharer 2003b) y con otros centros en la región de Puebla. Esta misma área de México Central fue el origen de muchos grupos cerámicos de comercio y de la distintiva obsidiana verde, bienes que se preferían como elementos de prestigio por la nobleza del Clásico Temprano maya. Estos centros distantes estaban en contacto con comunidades como Kaminaljuyú en las Tierras Altas mayas y está claro que también estaban en contacto con Copán (Sharer 2003b; Bell et al. 2004; Buikstra et al. 2004). Estas conexiones comerciales, subrayadas por las referencias visuales en la arquitectura, aumentaron el poder económico y el prestigio político del Fundador.

Las estructuras residenciales grandes del complejo palaciego real estaban rodeadas inicialmente por muros perimetrales construidos de tierra y guijarros (Sedat y López 2004; Traxler 2003, 2004b). Estos muros limitaban el acceso pero no la visibilidad a, y a través, de los grupos de edificios. Durante el reinado del Gobernante 2-el hijo de K'inich Yax K'uk' Mo' (según el texto en la Estela 63)—la definición del conjunto real se acentuó más. El extenso grupo residencial originalmente sobre la Plataforma Yune se reconstruyó sobre plataformas de albañilería cada vez más altas (Sharer et al. 1999, 2005). Esto levantó la residencia real sobre las plazas palaciegas adyacentes y mucho más sobre la Gran Plaza, sobre el Campo de Pelota I y sobre su santuario asociado, la Estructura Motmot. Finalmente, las plazas adyacentes también se levantaron (Traxler 2004b), junto con el santuario conocido como Estructura Papagayo. El conjunto del palacio real llegó a su máximo tamaño en esta ubicación y a su forma más restringida durante el reinado del quinto 0 del sexto gobernante de la dinastía de Copán. Ubicadas sobre plataformas de varios niveles, las grandes plazas en el precinto sur se colocaron sobre un nivel más alto, dominando grupos de plazas adicionales en terrazas más abajo.

El significado de estos grupos elaborados de plazas se encuentra en su contexto espacial, localizados en el corazón de la capital de la comunidad de Copán. Estos grupos fueron reconstruidos y expandidos en el curso del Clásico Temprano, según un patrón establecido en su fase inicial de construcción. Esto marca un fenómeno de conservación de construcción y refleja la importancia continua de estos grupos por múltiples generaciones. Comparaciones con complejos similares en otros sitios mayas sugieren que estos edificios facilitaban el espectro de las actividades residenciales y ceremoniales que se llevaban a cabo en el palacio real y, debido a esto, estos edificios se repitieron en por lo menos cuatro fases de construcción subsiguientes entre ca. 426 y 540 D.C. 
El contexto espacial de estos grupos de plazas palaciegas sugiere que sirvieron para mediar la comunicación entre el recinto real exclusivo sobre la cima de la Acrópolis y el resto del complejo arquitectónico en el centro de la comunidad copaneca (Traxler 2004b). Su ubicación-cerca de y conectados con la Acrópolis elevada, así como también con el Campo de Pelota y con los santuarios más al norte-sugiere que los habitantes participaban en las actividades de ambas áreas de la capital. En general, estos grupos de plazas fueron parte esencial del complejo palaciego real y el local para las actividades residenciales y para los asuntos administrativos de la familia extensa del rey y de su corte real.

\section{Los Palacios Reales dentro de la Capital del Clásico Temprano de Copán}

La arquitectura palaciega formaba una parte crucial del centro de Copán y el trabajo sin pago de la comunidad logró su construcción en secuencia y también su mantenimiento. Mientras que estos edificios seguramente servían como las residencias para la más alta élite, su ubicación central y su gran escala los hacía muy visibles a la comunidad. Estos edificios ciertamente proveían un recordatorio visual de la separación de la realeza y la jerarquía de privilegio elitista en la sociedad copaneca durante el período Clásico Temprano.

Una estructura de ritual bien conservada dentro de los grupos de albañilería de plaza fue la Estructura Perico. La ubicación no simétrica de su decoración de fachada sugiere que la visibilidad de su iconografía era importante para aquellos enfrente y al oeste del edificio. La altura de la Estructura Perico, incluyendo su probable techo abovedado y de peine, también sugiere que lo que se buscaba era que se viera y se reconociera fácilmente desde fuera de su plaza.

Considerando la visibilidad en general de los edificios dentro de estos grupos amontonados, el plan inicial de las fases tempranas permitía una visibilidad relativamente alta de un patio a otro, como también una visibilidad relativamente alta desde fuera hacia los grupos. Ya para las etapas tardías de estos grupos de plazas situadas sobre plataformas altas, comenzó una tendencia hacia reducir la visibilidad y a una mayor separación vertical. La mayor separación vertical del conjunto palaciego enfatizaba la altura como el señalador de los límites del palacio en vez de los muros. Los cambios en elevación en las terrazas de las plataformas subdividían los complejos en áreas y es posible que quizá también segregaran a los ocupantes en grupos separados. La expansión del complejo del palacio durante la parte temprana el siglo VI, para incluir un arreglo de plaza de dos niveles, puede 
que refleje una subdivisión aún más compleja, entre los habitantes del palacio, de la que existía anteriormente.

Vistos como arquitectura pública de control social, los edificios del palacio sí reflejan los intereses de la más alta élite por asegurar su posición y su autoridad dentro de la sociedad de Copán. Con el paso del tiempo, la posición social elevada de esta élite se manifestó más abundantemente por medio de la arquitectura físicamente elevada. Las plazas del palacio se volvían progresivamente más enfocadas a lo interior y su acceso más estrechamente controlado. Las expansiones durante la parte tardía del siglo $\mathrm{V}$, que crearon los Plazas $4 \mathrm{~B}$ y $4 \mathrm{C}$, mantuvieron, y más bien acrecentaron la separación entre la gente fuera del complejo del palacio y los de adentro. Con la construcción durante la parte temprana del siglo $\mathrm{VI}$, el proceso se repitió con edificios de plaza a una escala más grande ubicados sobre una plataforma expandida y elevada. Ya para mediados del siglo $\mathrm{VI}$ y para los reinados de los Gobernantes 7 y 8, las plazas del palacio se habían reubicado en otro punto, quizá donde había más espacio disponible para ensanchar la plaza, dejando la cima de la Acrópolis dominada por las versiones tempranas de los Patios Este y Oeste, que se ven hoy día.

Estas tendencias contrastan con el acceso relativamente abierto a la Gran Plaza del centro del sitio. Establecida alrededor del tiempo de la fundación de la dinastía y renovada con el pasar de los siglos que siguieron, la Gran Plaza continuó accesible a la mayoría de la sociedad de Copán y proveía un contraste al nivel más bajo de la topografía con la elevada Acrópolis, sus estructuras monumentales y con sus nobles ocupantes. Es de notar que la Acrópolis del VIII siglo mantuvo lados públicos con acceso visual y con graderías monumentales que llevaban del nivel de la plaza hasta los edificios sobre las Estructuras 10L-26 y 10L-11. Mientras a otros lados de este recinto real elevado los definían altas terrazas con acceso limitado y de poca visibilidad desde el nivel del suelo hacia las actividades o hacia los edificios que definían los patios del recinto real.

Los grupos de plazas del Clásico Temprano que se excavaron debajo de la superficie de la Acrópolis representan algunas de las muestras más tempranas y mejor conservadas de palacios reales mayas excavados hasta la fecha. Su documentación provee el fundamento para la evaluación y el análisis de esta secuencia arquitectónica sin precedentes. Estos grupos ofrecen evidencia crítica para dos de los más significantes hallazgos en la investigación reciente-el origen del estado en Copán y la fuerza de la organización sociopolítica de Copán durante el Período Clásico Temprano. 


\section{EL ORIGEN DEL ESTADO EN COPÁN}

La evidencia que proveen la excavación y la documentación del complejo palaciego debajo de la Acrópolis apoya la propuesta de que Copán se transformó y se mantuvo como la capital de una organización económica y política a nivel de estado durante el Período Clásico Temprano. Más específicamente, la evidencia actual apunta hacia K'inich Yax K'uk' Mo' como el agente de esta transformación inicial. Con su inauguración en el año 426 D.C. y su llegada a Copán como rey en el año 427 D.C., se instituyó un nuevo sistema político, importado muy posiblemente desde comunidades establecidas mucho antes en las Tierras Bajas mayas. Los datos arquitectónicos de la Acrópolis, combinados con evidencia obtenida de otras investigaciones en el sitio de Copán, como también aquellos datos del valle de Copán y alrededores, indican que los eventos que culminaron con la fundación de la dinastía establecieron a Copán como la capital de un estado secundario (Price 1978; Marcus 2004)-es decir, un sistema estatal derivado de un ejemplo más temprano de este complejo tipo de organización.

El texto sobre el Altar Q explícitamente dice que K'inich Yax K'uk' Mo' vino a Copán desde otro lugar en el año 427 D.C., pero desafortunadamente el texto no identifica el lugar de origen del Fundador. Varias fuentes de evidencia señalan su origen dentro de la región del Petén, en las Tierras Bajas Mayas Centrales (Sharer 2003a, 2003b, 2004; Buikstra et al. 2004), sugiriendo que la comunidad de Copán se convirtió en un estado secundario, formado o reformado bajo la influencia de los centros poderosos ubicados al norte (Marcus 2004). Referencias más tardías a K'inich Yax K'uk' Mo' lo llaman "Señor del Oeste", título también utilizado en los textos de Tikal (Martin y Grube 2000). La evidencia actual proveniente de los textos del período Clásico Temprano (Sharer 2000, 2004; Martin y Grube 2000), de los estilos en monumentos tempranos (Baudez 1983, 1994; Riese y Baudez 1983; Jones y Sharer 1980; Fash et al. 2004), de los restos arquitectónicos (Fash 1998; Sharer et al. 1999), y de las colecciones funerarias reales (Bell et al. 2004; Sharer 2003a, b), todos sugieren un papel primordial para Tikal en este proceso.

La cadena de evidencia que une a K'inich Yax K'uk' Mo' con las Tierras Bajas Mayas, y más específicamente con Tikal, comienza con la identificación de su lugar de entierro en la Tumba Hunal, descubierta por el PIAT en la base de la secuencia arquitectónica de la Acrópolis (Sharer 2004; Buikstra et al. 2004). La evidencia más directa viene de un artefacto de concha que se encontró en esta tumba y estaba decorado con un texto inciso que lee Yuh Wi' Te' ("su pendiente, raíz de árbol"). El título Wi' Te' se utilizaba exclusivamente para referirse al Fundador en Copán (Stuart 2004: 232). Pero esta referencia al Wi' Te' en el objeto que se encontró en la 
Tumba Hunal y la asociación exclusiva posterior en las inscripciones entre K'inich Yax K'uk' Mo' y un lugar llamado Wi' Te' Naah ("raíz árbol casa") es solamente una de muchas pistas que el entierro Hunal es el del Fundador (Sharer 2004).

Los materiales en la Tumba Hunal identifican al ocupante como un rey maya (Sharer y Traxler 2003). Análisis instrumentales de activación neutrónica realizados por Ronald Bishop, Dorie Reents-Budet y sus colegas identifican varias vasijas de la tumba como manufacturadas en Tikal, junto con otras de México Central y de las Tierras Altas mayas (Reents-Budet et al. 2004). Casi por 400 años después de que se cerró la Tumba Hunal, su ubicación continuó dedicada al fundador de la dinastía (Taube 2004). Una serie de templos funerarios superpuestos se construyeron sobre la Estructura Hunal, cada uno decorado con imágenes e iconografía recordando a K'inich Yax K'uk' Mo'.

Si toda esta evidencia identifica al ocupante de la Tumba Hunal como K'inich Yax K'uk' Mo', una serie de análisis de sus restos esqueléticos conducidos por Jane Buikstra y sus colegas pueden proveer la conexión crítica. Los análisis de isótopos de Estroncio y de Oxígeno indican que este individuo era originalmente de la región del Petén central, la ubicación de Tikal (Buikstra et al. 2004).

Durante el tiempo del establecimiento de la dinastía de Copán también abundan otras conexiones con Tikal. Las molduras de delantal en varias de las subestructuras más tempranas de albañilería en Copán se parecen al estilo arquitectónico prevaleciente durante el Clásico Temprano en Tikal y en sus centros subordinados. Los monumentos tempranos de Copán, incluyendo la Estela 35 y el Marcador Motmot, tienen numerosas conexiones estilísticas a la región del Petén y a Tikal (Fash et al. 2004; Baudez 1983). Los monumentos tempranos de Copán y de Quiriguá se han comparado por mucho tiempo con los de Tikal (Proskouriakoff 1950; Jones y Sharer 1980; Baudez 1983) y análisis recientes de las esculturas de Copán por Bárbara Fash (2004) se basa y avanza en estos estudios previos.

\section{Organización Sociopolítica en Copán durante el Clásico Temprano}

Los eventos de la llegada y el establecimiento instigados por K'inich Yax K'uk' Mo' claramente establecieron un nuevo estado maya con Copán como su capital. La investigación arqueológica apoya y amplifica nuestro conocimiento de estos eventos proporcionado por los textos históricos. Constante evidencia señala el cambio profundo en la organización sociopolítica de la comunidad de Copán y en el valle de Copán durante la parte temprana el siglo V (Fash 2005; Sharer et al. 2005; 
Marcus 2004). Evidencia tanto de Copán (Fash y Sharer 1991) como de centros más pequeños dentro de la comunidad de Copán (Canuto 2002, 2004) sugiere que K'inich Yax K'uk' Mo' estableció una estructura jerárquica de control político sobre una extensa área (Marcus 1993), que incluía el sitio de Quiriguá y su acceso a los recursos de jade del Río Motagua (Sharer 2002). Los siglos V y VI experimentaron un incremento en la población y en el número de asentamientos en el valle de Copán (Fash 1983) y la densa agrupación de asentamiento alrededor de la capital refleja la centralización del poder religioso y político allí (Fash 1991).

Los resultados de las excavaciones recientes en la Acrópolis documentan otro mecanismo de dominio político que contribuyó con el poder de los gobernantes de Copán. La arquitectura del centro de la comunidad representaba el control de la mano de obra y de los recursos que se utilizaron para construir tanto las instalaciones públicas como el complejo residencial privado del gobernante. La arquitectura de la era del establecimiento de la dinastía demuestra un cambio significativo en la escala y en el gasto de construcción (Carrelli 1997, 2004) como también demuestra un movimiento hacia la restricción de áreas del centro de la comunidad con muros perimetrales y con plataformas altas. Estos cambios en lo que se construyó y en la forma en que se diseñó el centro reflejan los intereses de los que tomaban las decisiones-el gobernante y su élite subordinada-quienes controlaban la comunidad de Copán.

La arquitectura monumental de la Acrópolis temprana requirió una fuerza laboral mucho más grande y también requirió más días de trabajo de los que se utilizaron en generaciones previas para erigir nuevos edificios en el centro de la comunidad. El tipo de arquitectura construida también demandaba materiales diferentes, más procesamiento y mano de obra más especializada que para construir los edificios más tempranos. Aun dentro de las largas tradiciones arquitectónicas establecidas, las plataformas más altas de tierra demandaban material de adobe más purificado, un tiempo más amplio de construcción y probablemente diseños repasados para poder obtener una estructura estable y durable. Era necesaria una gama más amplia de habilidades para la elaborada arquitectura de albañilería que se construyó durante el reinado de K'inich Yax K'uk' Mo' y el Gobernante 2. Estructuras altas de albañilería con fachadas complejas y con decoración modelada de estuco no se habían construido en Copán antes del establecimiento de la dinastía. La mano de obra necesaria frecuentemente se obtenía dependiendo del número de plataformas y de estructuras que se erigían en sucesión rápida por los reyes dinásticos iniciales (Sharer et al. 2005). 
La construcción de la arquitectura residencial del gobernante también necesitó una inversión significante de labor de la comunidad. El complejo del palacio real de los reyes tempranos fue una gran y costosa construcción. Los cálculos de Christine Carrelli (2004) para el primer siglo de construcción de la Acrópolis temprana revelan que durante el reino de K'inich Yax K'uk' Mo' se utilizaron más de 175,000 horas-hombre de labor en la plataforma y edificios iniciales del nuevo complejo real. Dados los estimados de población para este intervalo (Webster et al. 1992), cada adulto masculino en el valle de Copán tendría que haber trabajado un mes cada año del reino de K'inich Yax K'uk' Mo' para construir este complejo. Además, el estudio de Carrelli indica que las necesidades de trabajo para la construcción de la Acrópolis temprana, durante los primeros cinco años del reino del Gobernante 2, fueron aun mayores que estos totales.

La arquitectura majestuosa del Clásico Temprano de Copán, que se ha documentado en las excavaciones de la Acrópolis, requirieron los esfuerzos de muchos más trabajadores, más tiempo y más materiales de lo que requirieron las casas de cualesquier otros residentes de la élite. La disparidad entre el complejo de la nobleza y otras residencias de la élite, así como la utilización de la mano de obra de la comunidad para la construcción del complejo del palacio noble señalan una forma distinta de organización política y un nivel mucho más amplio de control político manejado por K'inich Yax K'uk' Mo' y el Gobernante 2. En su valoración en cuanto al desarrollo sociopolítico de Kaminaljuyú, Sanders (1974) observó hace mucho tiempo esta clase de disparidad en cuanto a construcción residencial y la utilización de la mano de obra de la comunidad para la construcción de la residencia monumental del gobernante. Él argumentó que estas disparidades, entre otros cambios que se observaron en Kaminaljuyú durante los períodos del Preclásico Tardío hasta el Clásico Temprano, eran indicadores de una forma estatal de organización política que controlaba a la comunidad. Flannery (1998) ha aplicado esta misma lógica para ilustrar el patrón de los orígenes del estado dentro de Mesoamérica y otras áreas, identificando el aparecimiento de palacios como un indicador crítico del desarrollo de un nivel estatal.

Durante el Período Clásico Temprano, Copán no era la capital urbana densamente poblada en lo que se convertiría años más tarde. Sin embargo, controlaba una comunidad extensa, incluyendo a Quiriguá (Marcus 1993; Sharer 1978) y a otros centros secundarios (Canuto 2002; Fash 2005) y controlaba engranajes comerciales de gran alcance e interacción de élites con sitios que incluían a Kaminaljuyú, Tikal y más allá (Fash 1991; Sharer 2002a, b). Para manejar esta empresa requería 
de una organización económica y política efectiva y manejada por una jerarquía administrativa no evidente antes del año 427 A.C. Parece cierto, entonces, que el establecimiento de la dinastía incluyó mucho más que simplemente la llegada de K'inich Yax K'uk' Mo'. Seguramente fue acompañado por un séquito de confianza de oficiales, guerreros, artesanos y otros especialistas, trayendo con ellos el bagaje de operaciones políticas y económicas del estado maya de las Tierras Bajas del Clásico Temprano (Traxler 2001). Entre los componentes de las operaciones estatales el centro comunal de Copán se rediseñó, estableciendo una gran plaza pública, un grupo de campo de pelota para celebrar el poder de los reyes divinos y un complejo de palacios para la nobleza, para el rey y su corte.

El interés en documentar estas muestras de la arquitectura del período Clásico Temprano va más allá de la comprensión de aspectos particulares de la historia arquitectónica o aun en cuanto al aspecto del tiempo necesario para el desarrollo de la organización estatal sociopolítica en Copán. El análisis de los grupos de palacios reales de Copán como parte del sistema total del engranaje de actividades (siguiendo a Rapoport 1990) nos provee otra manera de examinar la sociedad de Copán, su naturaleza y su estructura a través del tiempo. Estos complejos arquitectónicos formaron parte de un centro más grande y sirvieron como instalaciones donde se llevaron a cabo una amplia gama de actividades. La importancia del complejo real del palacio para esas actividades y para Copán como capital de una comunidad se enfatiza por la repetida construcción en el mismo lugar y con la misma organización a lo largo de todo el período Clásico Temprano.

Tal como se anotó antes, el momento de su aparición tiene implicaciones para nuestra comprensión en cuanto a la evolución de la comunidad copaneca, especialmente la importancia de los complejos palaciegos como indicadores de formas de estado de organización política. La inversión de recursos públicos en la construcción y en le mantenimiento de los palacios reales apunta hacia la consolidación estatal del poder político (Flannery 1998; Marcus 1995; Sanders 1974). Más allá de esto, un palacio real era el lugar para actividades de la vida doméstica, el manejo de los recursos de la comunidad, la administración de asuntos locales y distantes y el involucramiento social de élites y visitantes de la comunidad. Reflejaba una gran disparidad en cuanto a la inversión de recursos en su creación y mantenimiento tanto para la arquitectura como para sus habitantes. Todos estos factores le permitieron identificar y simbolizar a la nobleza y a su comunidad simultáneamente. Su estabilidad a lo largo del tiempo reforzó esta cercana identidad y simbolismo. 
La arquitectura real del Clásico Temprano de Copán, ya por mucho tiempo enterrada por las expansiones de la Acrópolis, se ha documentado por medio de una extensa red de excavaciones por medio de túneles. Como resultado de estas investigaciones, ahora se reconoce la importancia de esta secuencia de complejos palaciegos reales y su papel dentro del centro de la comunidad de Copán. Esta arquitectura monumental elaborada refleja las diversas tradiciones de Copán, sus transformaciones dramáticas con el establecimiento de la dinastía Clásica y el forjamiento de la organización estatal temprana en el siglo V. Las investigaciones arqueológicas han revelado la importancia de esta secuencia de palacios reales y su papel dentro de la capital de la comunidad como manifestaciones del poder de los reyes copanecos del Clásico Temprano.

\section{BIBLIOGRAFIA}

- Andrews, G. F. (1975). Maya Cities: Placemaking And Urbanization. Norman: University of Oklahoma Press.

- Baudez, C. F. (1994). Maya Sculpture of Copan: The Iconography. Norman: University of Oklahoma Press.

- Baudez, C. F. (ed.). (1983). Introducción a laArqueología de Copán, Honduras, Tomos I, II, III. Tegucigalpa: Proyecto Arqueológico Copán, Secretaría de Estado en el Despacho de Cultura y Turismo.

- Bell, E: E., Canuto M.A., Sharer R.J. (eds.) (2004). Understanding Early Classic Copan. Philadelphia: University of Pennsylvania Museum.

- Buikstra, J. E., Douglas Price T., Wright L.E., Burton J.H. (2004). Tombs from the Copan Acropolis: A Life History Approach. En Understanding Early Classic Copan, editado por E. Bell, M. Canuto y R. Sharer, pp. 191-212. Philadelphia: University of Pennsylvania Museum.

- Canuto, M. A. (2002). A Tale of Two Communities: Social and Political Transformation in the Hinterlands of the Maya Polity of Copan. Disertación Doctoral, Department of Anthropology, University of Pennsylvania.

- Canuto, M. A (2004). The Rural Settlement of Copan: Changes through the Early Classic. En E. Bell, M. Canuto y R. Sharer, Understanding Early Classic Copan (págs. 29-50). Philadelphia: University of Pennsylvania Museum. 
- Carrelli, C. W. (1997). Análisis Preliminar de la Construcción de la Acrópolis de Copan en el Clásico Temprano. Yaxkin 16:16-23.

- Carrelli, C. W. (2004). Measures of Power: The Energetics of Royal Construction at Early Classic Copan. En E. Bell, M. Canuto y R. Sharer, Understanding Early Classic Copan (págs. 113-127. Philadelphia: University of Pennsylvania Museum.

- Cheek, C. D. (1983) Excavaciones en la Plaza Principal. En Introducción a la Arqueología de Copán, Honduras, Tomo II, editado por C. F. Baudez, pp. 191-289. Tegucigalpa: Proyecto Arqueológico Copán, Secretaría de Estado en el Despacho de Cultura y Turismo.

- Cheek, C. D., Kennedy Embree V. (1983). Estructura 10L-2. En Introducción a la Arqueología de Copán, Honduras, Tomo II, editado por C. F. Baudez, pp. 93-141. Tegucigalpa: Proyecto Arqueológico Copán, Secretaría de Estado en el Despacho de Cultura y Turismo.

- Cheek C. D., Milla Villeda D.E. (1983). Estructura 10L-4. En Introducción a la Arqueología de Copán, Honduras, Tomo II, editado por C. F. Baudez, pp. 37-91. Tegucigalpa: Proyecto Arqueológico Copán, Secretaría de Estado en el Despacho de Cultura y Turismo.

- Fash, B. W. (2004). Early Classic Sculptural Development at Copan. En Understanding Early Classic Copan, editado por E. Bell, M. Canuto y R. Sharer, pp. 249-264. Philadelphia: University of Pennsylvania Museum.

- Fash, W. L. (1983). Maya State Formation: A Case Study and Its Implications. Disertación Doctoral, Department of Anthropology, Harvard University.

- Fash, W. L. (1991). Scribes, Warriors, and Kings: The City of Copan and the Ancient Maya. London: Thames and Hudson.

- Fash, W. L. (1998) Dynastic Architectural Programs: Intention and Design in Classic Maya Buildings at Copan and Other Sites. En S.D. Houston, Function and Meaning in Classic Maya Architecture (págs.223-270). Washington, DC: Dumbarton Oaks.

- Fash, W. L. (2001) Scribes, Warriors and Kings: The City of Copan and the Ancient Maya, edición revisada, London: Thames and Hudson. 
- Fash W. L., Fash B.W. (2000) Teotihuacan and the Maya: A Classic Heritage. En D. Carrasco, L. Jones y S. Sessions, Mesoamerica's Classic Heritage: From Teotihuacan to the Aztecs, (págs. 433-464). Boulder: University Press of Colorado.

- Fash, W. L., Sharer R.J. (1991) Sociopolitical Developments and Methodological Issues at Copan, Honduras: A Conjunctive Perspective. Latin American Antiquity 2:166-187.

- Fash W. L., Fash B.W., Davis-Salazar K. (2004) Setting the Stage: Origins of the Hieroglyphic Stairway Plaza on the Great Period Ending. En E. Bell, M. Canuto y R. Sharer, Understanding Early Classic Copan (págs. 65-83). Philadelphia: University of Pennsylvania Museum.

- Flannery, K. V. (1998) . The Ground Plans of Archaic States. En Archaic States, editado por G. M. Feinman y J. Marcus, pp. 15-57. Santa Fe, NM: School of American Research Press.

- Hall J., Viel R. (2004) The Early Classic Copan Landscape: A view from the PreClassic. En Understanding Early Classic Copan, editado por E. Bell, M. Canuto y R. Sharer, pp. 17-28. Philadelphia: University of Pennsylvania Museum.

- Harrison, P.D. (2003) Palaces of The Royal Court At Tikal. En Maya Palaces and Elite Residences, An Interdisciplinary Approach, J. Christie, editor, pp. 98119. Austin: University of Texas Press.

- Jones C., Sharer R.J. (1980) Archaeological Investigations in the Site Core of Quirigua. Expedition 23(1):11-19.

- Looper, M. G. (1999) New Perspectives on the Late Classic Political History of Quirigua, Guatemala. Ancient Mesoamerica 10:263-280.

- Maca, A. L. (2002) Spatio-Temporal Boundaries In Classic Maya Settlement Systems: Copan's Urban Foothills And The Excavations At Group 9J-5. Disertación Doctoral, Department of Anthropology, Harvard University.

- Marcus, J. (1993) Ancient Maya Political Organization. En Lowland Maya Civilization in the Eighth Century A. D., editado por J. A. Sabloff y J. S. Henderson, pp. 111-183. Washington, DC: Dumbarton Oaks. 
- Marcus, J. (1995) Where is Lowland Maya Archaeology Headed? Journal of Archaeological Research 3:3-35.

- Marcus, J. (2004) Primary and Secondary State Formation in Southern Mesoamerica. En Understanding Early Classic Copan, editado por E. Bell, M. Canuto y R. Sharer, pp. 357-373. Philadelphia: University of Pennsylvania Museum.

- Martin S., Grube N. (2000) Chronicle of Maya Kings and Queens. London: Thames and Hudson.

- Miller, J.C. (sf). Dissertación Doctoral, Department of Anthropology, University of Pennsylvania.

- Price, B. J. (1978) Secondary State Formation: An Explanatory Model. En Origins of the State: The Anthropology of Political Evolution, editado por R. Cohen y E. R. Service, pp. 161-186. Philadelphia, PA: Institute for the Study of Human Issues.

- Proskouriakoff, T. (1950) A Study of Classic Maya Sculpture. Publication 593. Washington, DC: Carnegie Institution of Washington.

- Rapoport, A. (1990) System of Activities and System of Settings. En Domestic Architecture and the Use of Space: An Interdisciplinary Cross-Cultural Study, editado por S. Kent, pp. 9-20. Cambridge: Cambridge University Press.

- Reents-Budet D., Ball J.W., Bishop R.L., Fields V., MacLeod B. (1994) Painting the Maya Universe: Royal Ceramics of the Classic Period. Durham, NC: Duke University Press.

- Riese B., Baudez C.F. (1983) . Esculturas de las Estructuras 10L-2 y 4. En Introducción a la Arqueología de Copán, Honduras, Tomo II, pp. 143-190, editado por C. F. Baudez. Tegucigalpa: Proyecto Arqueológico Copán, Secretaría de Estado en el Despacho de Cultura y Turismo.

- Sanders, W. T. (1974) From Chiefdom to State: Political Evolution at Kaminaljuyu, Guatemala. En Reconstructing Complex Societies, editado por Charlotte B. Moore. Supplement to the Bulletin of the American Schools of Oriental Research 20:97-116. 
- Sedat D. W., López F. (2004) Initial Stages in the Formation of the Copan Acropolis. En Understanding Early Classic Copan, editado por E. Bell, M. Canuto y R. Sharer, pp. 85-99. Philadelphia: University of Pennsylvania Museum.

- Sharer R. J. (1978) Summary of Architecture and Constructional Activity. En The Prehistory of Chalchuapa, El Salvador, vol. 1, editado por R. J. Sharer, pp. 121-132. Philadelphia: University of Pennsylvania Press.

- Sharer R. J. (1997) Political and Ideological Power and the Origins of the Acropolis. ECAP Paper No. 1. Philadelphia, PA: Instituto Hondureño de Antropología e Historia y el University of Pennsylvania Museum Early Copan Acropolis Program.

- Sharer R. J. (2002) Early Classic Dynastic Origins in the Southeastern Maya Lowlands. En Incidents of Archaeology in Central America and Yucatan: Essays in Honor of Edwin M. Shook, editado por M. Love, M. Poponoe de Hatch y H. Escobedo, pp. 459-476. Lanham MD: University Press of America.

- Sharer R. J. (2003a) Founding Events and Teotihuacan Connections at Copan, Honduras. En Teotihuacan and the Maya: Reinterpreting Early Classic Interaction, editado por G. Braswell. Austin: University of Texas Press

- Sharer R. J. (2003b) Tikal and the Copan Dynastic Founding. En Tikal: Dynasties, Foreigners, \& Affairs of State: Advancing Maya Archaeology, editado por J. A. Sabloff, pp. 319-353. Santa Fe, NM: SAR.

- Sharer R. J. (2004) External Interaction at Early Classic Copan. En Understanding Early Classic Copan, editado por E. Bell, M. Canuto y R. Sharer, pp. 297-317. Philadelphia: University of Pennsylvania Museum.

- Sharer R. J., Miller J.C., Traxler L.P. (1992) Evolution of Classic Period Architecture in the Early Acropolis, Copan: A Progress Report. Ancient Mesoamerica 3:145-159.

- Sharer R.J., Traxler L.P. (2003) Las Tumbas Reales Más Tempranas de Copán: Muerte y Renacimiento en un Reino Maya Clasíco. En Antropología de la Eternidad. La Muerte en la Cultura Maya, editado por A. Cuidad Ruiz, M. H. Ruz Sosa y M. J. Iglesias Ponce de León. Sociedad Española de Estudios Mayas y del Centro de Estudios Mayas, Instituto de Investigaciones Filológicas, UNAM. 
- $\quad$ Sharer, R.J., Traxler L.P., Sedat D.W., Bell E.E., Canuto M.A., Powel C. (1999). Early Classic Architecture beneath the Copan Acropolis: A Research Update. Ancient Mesoamerica 10:3-23.

- Sharer, R. J., Sedat D.W., Traxler L.P., Miller J.C., Bell E.E. (2005). $\quad$ E a r I y Classic Royal Power in Copan: The Origins and Development of the Acropolis (ca. AD 250-600). En Copan: The History of an Ancient Maya Kingdom, editado por E. W. Andrews y W. L. Fash. Santa Fe: School of American Research Press.

- Strömsvik, Gustav. (1952). The Ball Courts at Copan, with Notes on Courts at La Unión, Quirigua, San Pedro Pinula, and Asunción Mita. Contributions to American Anthropology and History No. 55. Washington, DC: Carnegie Institution of Washington.

- Taube, Karl. (2004). Structure 10L-16 and Its Early Classic Antecedents: Fire and the Evocation and Resurrection of K'inich Yax K'uk' Mo'. En Understanding Early Classic Copan, editado po r E. Bell, M. Canuto y R. Sharer, pp. 265-295. Philadelphia: University of Pennsylvania Museum.

- Traxler, Loa P. (2001). The Royal Court of Early Classic Copan. En Royal Courts of the Ancient Maya, vol. 2, editado por T. Inomata y S. D. Houston, pp. 47-73. Boulder, CO: Westview Press.

- Traxler, Loa P. (2003). At Court in Copan: Palace Groups of the Early Classic. En Maya Palaces and Elite Residences, editado por J. Christie, pp. 46-68 Austin: University of Texas Press.

- Traxler, Loa P. (2004a). Redesigning Copan: Early Architecture of the Polity Center. En Understanding Early Classic Copan, editado por E. Bell, M. Canuto y R. Sharer, pp. 65-83. Philadelphia: University of Pennsylvania Museum.

- Traxler, Loa P. (2004b). Evolution and Social Meaning of Patio and Courtyard Group Architecture of the Early Classic Acropolis, Copan, Honduras. Disertación Doctoral, Department of Anthropology, University of Pennsylvania, Philadelphia.

- Viel, René. (1999). The Pectorals of Altar Q and Structure 11: An Interpretation of the Political Organization at Copan, Honduras. Latin American Antiquity 10(4):377-399.

- Webster, David, William T. Sanders y Peter van Rossum. (1992). A Simulation of Copan Population History and its Implications. Ancient Mesoamerica 3:185197. 